\title{
COMPACTIFICATION WITH SCALAR FIELDS
}

\author{
J.-M. Gérard $\left.{ }^{*}\right)$, Jihn E. Kim ${ }^{+}$and H.P. Nilles $\left.{ }^{\ddagger}\right)$ \\ CERN -- Geneva
}

\begin{abstract}
$\underline{\text { ABSTRACT }}$
A role of scalar fields in compactification is emphasized. A scale invariant six-dimensional interacting scalar field theory is studied in some detail. Compactification occurs even at tree level, and we obtain a relation between the compactification size (a), vacuum expectation values of scalar fields (v) and the $\lambda \phi^{3}$ interaction strength, $a^{2} v \lambda=0(1)$. We also point out some subtleties in the derivation of the four-dimensional gauge symmetry in this context.
\end{abstract}

*) IISN Fellow, on leave of absence from Institut de Physique Théorique, Université Catholique de Louvain (Belgium).

+ ) Permanent address after July 1984: Dept. of Physics, Seoul National University, Seoul 151, Korea.

\#) Permanent address: Département de Physique Théorique, Université de Genève, 1211 Geneva 4, Switzerland. Partially supported by the Swiss National Science Foundation. 
One of the utmost important questions in Kaluza-Klein theories is the compactification of extra dimensions. This mechanism provides a rationale for a physical study of extended space-time dimensions.

In the literature ${ }^{1}$, roles of gauge fields in spontaneous compactification at the classical level have been extensively discussed. The introduction of these extra gauge fields is a drawback in the Kaluza-Klein philosophy ${ }^{2}$ ), where it was originally hoped that the observed gauge interactions are remnants of compactification processes of a higher dimensional gravity. If the original Kaluza-Klein idea is not achievable in its pure form, roads to possible generalizations are widely open. A few have been considered 3 ) in connection with the fermion quantum number problem.

In this paper, we study a possible role of scalar fields in the compactification process. A simple and non-trivial example is that of scalar fields interacting in six dimensions. For definiteness, we take a "scale invariant" action of scalars and metric fields, *

$$
\begin{aligned}
S & =\int d^{b} x \sqrt{-g}\left(\frac{\varepsilon}{2} \phi^{a} \phi^{a} R\right. \\
& -\frac{1}{2} g^{M N} \partial_{M} \phi^{a} \partial_{N} \phi^{a}-\frac{\lambda}{3 !} d^{a b c} \phi^{a} \phi^{b} \phi^{c} \\
& \left.+\alpha_{0}\right)
\end{aligned}
$$

where $\varepsilon$ is a positive dimensionless parameter, a,b,c are internal symmetry group indices of scalar fields, and $M, N, P, Q$, etc., are six-dimensional Einstein indices. $\ell_{0}$ is the free Lagrangian for massless fermions and additional massless scalars. We will not consider cubic curvature terms, because the action $S$ alone will show the desired properties.

In $D$ dimensions, bosons ( $\phi)$, fermions $(\psi$ ) and curvature (e.g., R, etc.) fields carry $(D-2) / 2,(D-1) / 2$ and 2 energy dimensions respectively. Therefore, scale invariant scalar self-interactions can be introduced only in

(*) The signature is $(-+++++)$ and our sign convention is given by $\mathrm{R}_{\mu \nu} \sim \Gamma_{\mu \nu, \lambda}^{\lambda}-\dot{\Gamma}_{\mu \lambda, \nu}^{\lambda}$. 
$D=4$ and 6 . In higher dimensions, our scale invariant $\lambda \phi^{3}$ term could be replaced by terms of higher order in $R, e \cdot g \cdot, R^{D / 2}$. In passing, we remark that our example is the remaining possibility for induced gravity ${ }^{4}$ in asymptotically free field theory.

Even though the action (1) has these beautiful properties, it may fall short of describing the real world because there are not enough extra dimensions, or the observed gauge fields are composite in nature coming from the $\ell_{0}$ part due to the compactification process. Now let us go back to (I).

We are sure that we live in a four-dimensional Minkowski space here and now. The extra two dimensions must be compactified. With only one scalar field, the $\lambda \phi^{3}$ interaction does not provide the desired compactification; we have to introduce more scalar fields, in such a way that a (symmetric) tensor, like $\left(\partial_{\mu} \phi^{a}\right)\left(\partial_{\nu} \phi^{a}\right)$, for example, receives a vacuum expectation value. An $S U(N)$ global symmetry is imposed in order to keep a unique coupling constant. For simplicity, we take scalars in one adjoint representation of $\mathrm{SU}(\mathrm{N})$. We know that in the flat limit this model is still asymptotically free for $N \geq 5$. For the compactification purpose, however, it is enough to study a simpler group, SU(3). Certainly, the dynamics underlying the compactifcation with SU(3) will not be destroyed with a larger group.

The specific example Eq. (1) is fundamentally different from the other examples of compactification ${ }^{1}$ ) in that we do not introduce any scale, for example a cosmological constant and/or a dimensional gauge coupling constant. Therefore, it is not a priori clear whether the compactification will occur. Our result will show that there exist compactification solutions with a scale introduced by dimensional transmutation.

The stationary action principle, with respect to the variations of the metric $g^{M N}$ and the scalar field $\phi^{a}$, leads to generalized Einstein and field equations respectively:

$$
\begin{aligned}
& \varepsilon \phi^{a} \phi^{a}\left(R_{M N}-\frac{1}{2} R g_{M N}\right) \\
& =T_{M N}+\varepsilon\left[\left(\phi^{a} \phi^{a}\right)_{i M N N}-g_{M N}\left(\phi^{a} \phi^{a}\right)_{i P}^{i P}\right]
\end{aligned}
$$


$-3-$

$\phi_{i P}^{a} i P=-\varepsilon R \phi^{a}+\frac{\partial V}{\partial \phi^{a}}$

where $\mathrm{T}_{\mathrm{MN}}$ is the energy-momentum tensor

$$
T_{M N}=\phi_{i M}^{a} \phi_{i N}^{a}-g_{M N}\left(\frac{1}{2} \phi^{a-P_{i}} \phi_{i p}^{a}+V\right)
$$

and $V$ is the potential

$$
V=\frac{\lambda}{6} d^{a b c} \phi^{a} \phi^{b} \phi^{c}
$$

The last term of Eq. (2) comes from variation of $\mathrm{R}_{M N}$, which gives a vanishing contribution for the case of Einstein-Hilbert action. Let us search for solutions of the above equations. Certainly, $\phi^{a}=C^{a}$ (constant) are unstable solutions of Eq. (2) and (3), which does not shed any light on the present world. We look for a maximally symmetric compactification soltron:

$$
M^{6} \longrightarrow M^{4} \times S^{2}
$$

with non-vanishing vacuum expectation values of scalar fields to generate the Planck mass dynamically. 
- 4 -

Contracting indices of $\mathrm{Eq} \cdot(2)$, we obtain

$$
\begin{aligned}
\varepsilon R \phi^{a} \phi^{a} & =\phi_{i p}^{a} \phi^{a i p} \\
& +3 V+\frac{5}{2} \varepsilon\left(\phi^{a} \phi^{a}\right)_{i p}^{i p}
\end{aligned}
$$

On the other hand, multiplying Eq. (3) by $\phi^{a}$, we obtain:

$$
\begin{aligned}
& \varepsilon R \phi^{a} \phi^{a}=\phi_{i P}^{a} \phi^{a} ; P \\
& +3 V-\frac{1}{2}\left(\phi^{a} \phi^{a}\right)^{i P} ; P
\end{aligned}
$$

From Eqs. (7) and ( 8 ), we conclude a consistency equation

$$
(5 \varepsilon+1)\left(\phi^{a} \phi^{a}\right)_{i p}^{i p}=0
$$

The solution $\varepsilon=-(D-2) /(4 D-4)=-1 / 5$ corresponds to a conformally invarant $D=6$ theory with a wrong sign for the Newton constant $G_{N}$. In the remainder of the paper, we therefore satisfy

$$
\left(\phi^{a} \phi^{a}\right)_{i p}^{i p}=0
$$

This implies that the value of the action at a stationary point is

$$
\langle S\rangle=\frac{1}{2} \int d^{6} x \sqrt{-g}\langle V\rangle
$$


$-5-$

We look for classical solutions of $g_{M N}$ and $\phi^{a}$ on the manifold $M^{4} \times s^{2}$. The $x^{5}$ and $x^{6}$ coordinates being curled up, we introduce the following ansatz for the metric

$$
d s^{2}=\eta_{\mu \nu} d x^{\mu} d x^{\nu}+a^{2}\left(d \theta^{2}+\sin ^{2} \theta d \varphi^{2}\right)
$$

where $a$ is the compactification radius related to the inverse of the vacuum expectation value of the scalar fields. Let us consider the scalar octet of SU (3), $\Phi=\lambda^{a} \phi^{a}$, where $\lambda^{a}(a=1,2, \ldots, 8)$ are the usual Gell-Mann matrices. The two independent $s^{2}$ invariant monopole-like solutions ${ }^{5)}$ are

(A) $\Phi=v \hat{r}_{i} \lambda_{i}+\omega \lambda_{8}$

(8) $\Phi_{i j}=i \tilde{v} \varepsilon_{i j k} \hat{r}_{k}+\tilde{w}\left(\hat{r}_{i} \hat{r}_{j}-\frac{1}{3} \delta_{i j}\right)$

where cases $(\mathrm{A})$ and $(\mathrm{B})$ provide $\mathrm{SU}(3) \rightarrow \mathrm{SU}(2) \times \mathrm{U}(1)$ and $\mathrm{SU}(3) \rightarrow \mathrm{SO}(3)$ symmetry breakings respectively. The unit vector $\hat{\mathrm{r}}$ is

$$
\begin{aligned}
\hat{r} & =\left(\hat{r}_{1}, \hat{r}_{2}, \hat{r}_{3}\right) \\
& =(\sin \theta \cos \varphi, \sin \theta \sin \varphi, \cos \theta)
\end{aligned}
$$

$(15)$

These solutions obviously satisfy the constraint (10). Now the basic aquatrons (2) and (3) reduce to

$$
\varepsilon \phi^{a} \phi^{a} R^{(4)}=2\langle V\rangle=0
$$

(16) 
- $6-$

$$
\begin{gathered}
\varepsilon \phi^{a} \phi^{a} R^{(2)}=\phi_{i p}^{a} \phi^{a} ; P \\
\frac{1}{a^{2}}\left[\frac{\partial^{2}}{\partial \theta^{2}}+\frac{1}{\sin ^{2} \theta}\left(\frac{\partial^{2}}{\partial \varphi^{2}}+\sin \theta \cos \theta \frac{\partial}{\partial \theta}\right)\right] \phi^{a} \\
=-\varepsilon R^{(2)} \phi^{a}+\frac{\lambda}{2} d^{a b c} \phi^{a} \phi^{b} \phi^{c}
\end{gathered}
$$

where $R^{(4)}=0$ and $R^{(2)}=2 / a^{2}$ are the curvature scalars of four-dimensional Minkowski space and of the two-dimensional sphere respectively. It is worthwhile noting that compactification occurs into a Minkowski space for a vanishing value of the six-dimensional action [compare Eq. (11) with Eq. (16)].

We present solutions separately for cases (A) and (B).

Solution (A): $\phi^{a} \phi^{a}=v^{2}+w^{2}$

$$
\begin{aligned}
& V=\frac{\lambda}{6 \sqrt{3}} \omega\left(3 v^{2}-\omega^{2}\right) \\
& \frac{\partial V}{\partial \phi^{a}}=\frac{\lambda}{\sqrt{3}} \omega \phi^{a} \\
& \frac{\partial V}{\partial \phi^{8}}=\frac{\lambda}{2 \sqrt{3}}\left(v^{2}-\omega^{2}\right) \\
& \phi^{a ; P}=-\frac{2}{a^{2}} \phi^{a} \quad(a=1,2,3)
\end{aligned}
$$


$-7-$

Therefore, the field equations (16) - (18) lead to

$$
\begin{aligned}
\lambda a^{2} v & =-\frac{3}{2} \\
\varepsilon & =\frac{1}{4}
\end{aligned}
$$

where $\mathrm{w}^{2}=3 \mathrm{v}^{2}$.

Solution (B): $\quad \phi^{a} \phi^{a}=\tilde{v}^{2}+\tilde{w}^{2} / 3$

$$
\begin{aligned}
& V=\frac{\lambda}{6} \tilde{w}\left(\frac{1}{9} \tilde{w}^{2}-\tilde{v}^{2}\right) \\
& \frac{\partial V}{\partial \phi^{a}}=-\frac{\lambda}{3} \tilde{w} \phi^{a} \quad(a=2,5,7) \\
& \frac{\partial V}{\partial \phi^{a}}=\frac{\lambda}{2}\left(\frac{\tilde{w}}{3}-\frac{\tilde{v}^{2}}{\widetilde{w}}\right) \phi_{(a=1,3,4,6,8)}^{a} \\
& \phi_{i P}^{a ; P}=-\frac{2}{a^{2}} \phi^{a} \quad(a=2,5,7) \\
& \phi^{a ; P}=-\frac{6}{a^{2}} \phi^{a}(a=1,3,4,6,8)
\end{aligned}
$$


$-8-$

From these, we obtain

$$
\begin{aligned}
\lambda a^{2} \tilde{v} & =-3 \\
\varepsilon & =\frac{5}{2}
\end{aligned}
$$

where $\tilde{w}^{2}=9 \tilde{v}^{2}$.

The Planck mass squared or the inverse Newton constant $\mathrm{G}_{\mathrm{N}}^{-1}$ is obtained by performing $\theta$ and $\phi$ integration of Eq. (1) for the solutions (A) and (B). We obtain

$$
\begin{aligned}
& M_{P l}^{2}=\frac{48 \pi^{2}|v|}{\left|\lambda\left(M_{P l}\right)\right|} \text { for }(A) \\
& M_{P l}^{2}=\frac{960 \pi^{2}|\tilde{v}|}{\left|\lambda\left(M_{P l}\right)\right|} \text { for }(B)
\end{aligned}
$$

Because we did not introduce any scale in the beginning, Eqs. (32) and (33) are the definition of a scale. Then the compactification scale is logrithmically dependent upon the distance scale we probe,

$$
\begin{aligned}
a^{2} & =\frac{72 \pi^{2}}{\lambda \lambda\left(M_{P l}\right) M_{P l}^{2}} \\
a^{2} & =\frac{2880 \pi^{2}}{\lambda \lambda\left(M_{P l}\right) M_{P l}^{2}}
\end{aligned}
$$

for (A) (34)

for (B) (35) 
$-9-$

What is the gauge symmetry resulting from the topology of scalar fields given by (6)? One naively expects that an $S O(3)$ gauge symmetry survives the compactification. In the $(4+\mathrm{N})$-dimensional case, we could expect $\mathrm{SO}(\mathrm{N}+1)$. However, in the framework we consider, there is a clash between the local gauge symmetry associated with the global symmetry acting on the additional scalar fields $\phi^{a}$. We therefore expect a (partial) breakdown of the gauge group $\mathrm{SO}(\mathrm{N}+1)$, due to the nontrivial coupling induced through the kinetic term,

$$
-\frac{1}{2} \int d^{4} x \int d^{N} y \sqrt{-g} g^{P Q} \partial_{P} \phi^{a} \partial_{Q} \phi^{a}
$$

where we considered a $(4+N)$-dimensional theory. Expanding the fields $\phi^{a}$ on

$$
\begin{gathered}
\phi^{a}(x, y)=\sum_{\ell, m} \phi_{m}^{a l}(x) Y_{m}^{l}(y), \\
-l \leq m \leq l
\end{gathered}
$$

with the spherical harmonic function obeying the orthogonality relation

$$
\begin{aligned}
& \int d^{N} y \sqrt{g(y)} Y_{m}^{l}(y) Y_{m^{\prime}}^{\ell^{\prime}}(y)=\delta_{l \ell^{\prime}} \delta_{m m^{\prime}}{ }^{(38)} \\
& -\frac{1}{2} \int d^{4} x \sqrt{-g(x)} g^{\mu \nu} \sum_{l, m}\left(D_{\mu} \phi^{a \ell}\right)_{m}^{*}\left(D_{\nu} \phi^{a l}\right)_{m}{ }^{(39)}
\end{aligned}
$$


where the covariant derivative in the Minkowski space is given by

$$
\begin{aligned}
& \left(D_{\mu} \phi^{a l}\right)_{m}=\lambda_{\mu} \phi_{m}^{a l}(x)
\end{aligned}
$$

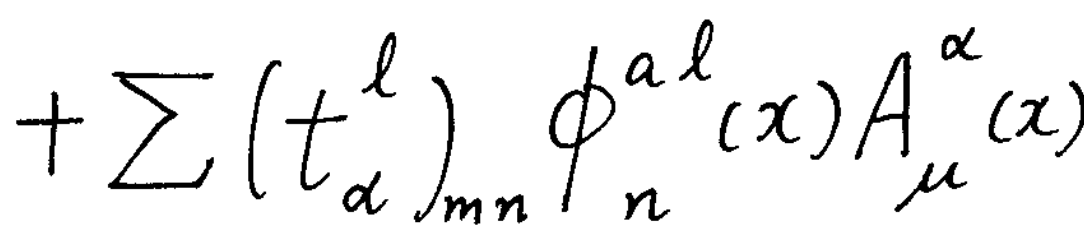

For example, if the non-vanishing vacuum expectation value of the scalar fields corresponds to $\ell=1$, the last term of Eq. (40) shows the mixing of $\mathrm{A}_{\mu}^{\alpha}[\alpha=1,2, \ldots, \mathrm{N}(\mathrm{N}+1) / 2]$ and $\phi^{\mathrm{al}}$, which render the partial breaking of the SO $(\mathrm{N}+1)$ gauge symmetry to $\mathrm{SO}(\mathrm{N})$. In the discussed solution (A), we thus remain with an $\mathrm{SO}(2)$ gauge symmetry.

In conclusion, we have shown explicitly the role of scalar fields participating in the compactification process. As an example, we considered the scale invariant action in six dimensions with $\lambda \phi^{3}$ interactions. One of the interesting results in this example is the relation between the vacuum expectation values of the scalar fields (identified as $\mathrm{M}_{\mathrm{Pl}}$ ) and the compactification size. If compactification is due to scalars, the resulting fourdimensional gauge symmetry is smaller than expected from the counting of the isometries of the compactified space. The creation of these symmetries already implies their partial breakdown.

\section{ACKNOWLEDGEMENTS}

We thank Sergio Ferrara, Peter Forgacs and Christof Wetterich for helpful discussions. 


\section{REFERENCES}

1) Z. Horvath, L. Palla, E. Cremmer and J. Scherk, Nuc1. Phys. B127 (1977) 57 ;

J.-F. Luciani, Nuc1. Phys. B135 (1978) 111;

P.G.0. Freund and M. Rubin, Phys. Lett. 97B (1980) 233;

S. Randjbar-Daemi, A. Salam and J. Strathdee, Nuc1. Phys. B214 (1983) 491 .

2) Th. Kaluza, Sitzungsber. Preuss. Akad. Wiss. Berlin, Math. Phys. KI (1921) 966;

0. Klein, Z. Phys. 37 (1926) 895;

B.S. De Witt, in "Relativity, Groups and Topology", B. De Witt and

C. De Witt, eds., (New York, Gordon Breach, 1964);

R. Kerner, Ann. Inst. H. Poincaré 9 (1968) 143;

Y.M. Cho and P.G.O Freund, Phys. Rev. D1.2 (1975) 1711;

L.N. Chang, K.I. Macrae and F. Mansouri, Phys. Rev. D13 (1976) 235;

E. Witten, Nucl. Phys. B186 (1981) 412.

3) E. Witten, Princeton University preprint "Fermion Quantum Numbers in Kaluza-Klein Theory" (1983);

S. Weinberg, Univ. of Texas preprint UTTG-1-84;

C. Wetterich, Univ, of Bern preprint BUTP-84/5 (1984).

4) A.D. Sakharov, Dok. Akad. Nauk. SSSR 177 (1967) 70 [Sov. Phys. Dok1. 12 (1968) 1040];

A. Zee, Phys. Rev. Lett. 42 (1979) 417;

L. Smolin, Nuc1. Phys. B160 (1979) 253;

S. Adler, Rev. Mod. Phys. 54 (1982) 729.

5) E. Corrigan, D. Fairlie, J. Nuyts and D. Olive, Nuc1. Phys. B106 (1976) 475.

6) See, for example:

S. Weinberg, Phys. Lett. 125B (1983) 265. 